\title{
Erratum: Fully Device-Independent Quantum Key Distribution [Phys. Rev. Lett. 113, 140501 (2014)]
}

\author{
Umesh Vazirani and Thomas Vidick \\ (Received 20 January 2016; published 23 February 2016)
}

DOI: 10.1103/PhysRevLett.116.089901

This Letter corrects numerical errors that were made in the derivation of the following explicit bound on the raw key rate as a function of the error rate, which appears at the top of the second column on p. 3:

$$
H_{\min }^{\varepsilon}(A \mid \mathcal{P E}) \geq-\frac{11}{3} \log \left(\frac{11}{12}+\frac{2}{3} \eta\right)
$$

where $\eta$ is the bit error rate. The bound (1) involves constants derived from a more precise and general bound which appears as Eq. (10) in the Supplemental Material to the Letter. The mistake can be traced to two simple numerical errors-an omitted square root and a factor 2 in the last centered equation in the proof of the "guessing lemma," Lemma 5 in the supplemental material to the Letter, which should read

$$
2-\frac{I^{2}}{4} \leq 4 \sqrt{2} \eta+24 \sqrt{2} \nu
$$

a bound which follows immediately from $I=2 \sqrt{2}-4 \eta-24 \nu$, as defined in the Supplemental Material to the Letter. This causes the conclusion of the lemma to get updated to

$$
\delta \geq\left(\frac{1}{2}-2^{1 / 4} \eta^{1 / 2}\right)-10 \nu^{1 / 2}
$$

Propagating the change to Eq. (10) in the proof of Theorem 1 from the Supplemental Material to the Letter, the corrected equation should read

$$
\kappa>-6\left(1-\tau^{\prime}\right) \log \left(\frac{11}{12}+\frac{3}{2^{1 / 4} \times 8} \sqrt{\frac{\eta}{(1-\tau)}}\right)-O\left(\frac{\log (1 / \varepsilon)}{\eta^{2} m}\right) .
$$

(Here, $m$ is the number of rounds of the protocol and $\kappa$ the smooth min-entropy rate of the raw key.) Any choice of $\tau, \tau^{\prime}$ in Eq. (2) such that $\tau+\tau^{\prime}>1$ leads to a valid bound, and the choice may be optimized as a function of $\eta$. A possible choice is $\tau=1 / 10$ and $\tau^{\prime}=91 / 100$, leading to the final bound

$$
H_{\min }^{\varepsilon}(A \mid \mathcal{P E}) \geq-\frac{54}{100} \log \left(\frac{11}{12}+\frac{\sqrt{10}}{8 \times 2^{1 / 4}} \sqrt{\eta}\right),
$$

which is the corrected form of Eq. (1).

The corrected Eq. (3) still shows that the protocol achieves a linear key rate in the presence of constant noise level (for quantum key distribution in the device-independent model of security), though the numerical bound is worse than originally claimed.

The error was brought to our attention by Erik Woodhead and independently by Zhu Cao and Xiongfeng Ma. 